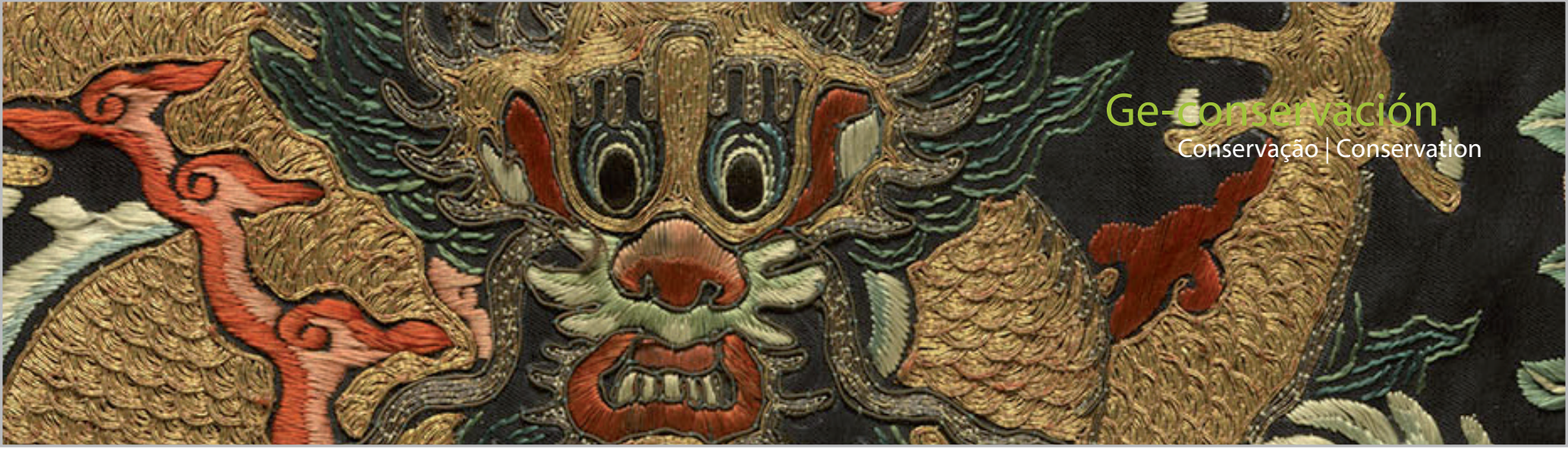

\title{
Estudio científico y tratamiento de conservación de un vestido Chino Jifu de finales del siglo XIX
}

\author{
César Rodríguez Salinas, Livio Ferrazza
}

\begin{abstract}
Resumen: La siguiente investigación aborda el estudio pormenorizado de un vestido oficial de la corte Qing también conocido como jifu. Perteneciente a la colección del Kunstmuseum Den Haag $(\mathrm{KMDH})$ en los Países Bajos, dicho vestido ha contribuido a interpretar y contextualizar el momento en el que el comercio de objetos textiles de Oriente se encontraba en pleno auge en Occidente a través del movimiento cultural conocido como Orientalismo. Originalmente confeccionado para ser vestido por un hombre de la corte, la prenda refleja en la actualidad la característica forma de un abrigo de mujer de finales de los años 20.
\end{abstract}

En el presente estudio, la aplicación de técnicas de análisis científico, a través de la colaboración del Instituto Valenciano de Conservación y Restauración e Investigación (IVCR+i), ayudó no solamente a determinar la calidad de los materiales presentes, sino también, a justificar los elementos de degradación activos en el momento de estudio del vestido. Todo ello, junto con el análisis de los cambios sufridos respecto al diseño original, ayudó a determinar el mejor tratamiento de conservación y restauración posible para salvaguardar tan preciado bien cultural.

Palabras clave: conservación y restauración de indumentaria histórica, Dinastía Qing, Dragon Robe, Jifu, orientalismo, seda cargada

\section{Conservation and Scientific research of a Chinese Jifu dress from the late XIX century}

Abstract: The following research reflects a thorough study of a dragon robe, also known as jifu, from the Kunstmuseum Den Haag collection (here after $\mathrm{KMDH}$ ) in the Netherlands. The dress -modified in the late $20^{\text {th }}$ century- is a consequence of the Chinese goods that became so fashionable after the end of the Qing Dynasty in 1911 in Europe and America. This new movement also known as Orientalism, brought a huge fanaticism to everything that was connected with the East, which it was reflected not only in art but also in Western fashion. Originally designed as a man attire worn solely by the emperor's circle, the object currently reflects a shape of a woman coat typically found during the late 20 's.

In order to know more about the materials used for the confection of the dress as well as to identify the degradation components presented, different analytical techniques were carried out by the international collaboration made with InstitutoValenciano de Conservacióny Restauración e Investigación (IVCR+i) in Spain. The results gathered from those analysis helped with the decision choice for the final conservation treatment.

Keywords: fashion conservation, Qing Dinasty, Dragon Robe, Jifu, orientalism, weight silk

\section{Estúdio científico e tratamento de conservação de um vestido Chino Jifu de finais do siglo XIX}

Resumo: A seguinte pesquisa aborda o estudo detalhado de um vestido oficial da corte Qing também conhecido como jifu. Pertencente à coleção do Kunstmuseum Den Haag (KMDH) nos Países Baixos, este vestuário contribuiu para interpretar e contextualizar o momento em que o comércio de têxteis do Oriente se encontrava em pleno desenvolvimento no Ocidente, através do movimento cultural conhecido como Orientalismo. Originalmente confecionado para ser vestido por um homem da corte, a roupa reflete na atualidade a forma de um casaco de mulher do final dos anos 20.

No presente estudo, a aplicação de técnicas de análise científica, através da colaboração do Instituto Valenciano de Conservación y Restauración e Investigación (IVCR+i), ajudou não só a determinar a qualidade dos materiais presentes, mas também para justificar os elementos de degradação ativos no momento do estudo do vestuário. Tudo isso, junto com a análise das mudanças sofridas em relação ao desenho original, ajudou a determinar o melhor tratamento de conservação e restauro possível para salvaguardar tão precioso bem cultural.

Palavras-chave: conservação e restauro de vestuário histórico, Dinastia Qing, Dragon Robe, Jifu, orientalismo, seda com carga 


\section{Introducción}

Con motivo de la exposición temporal "Global WardrobeDe wereldwijde modeconnectie", programada para el otoño del 2021 en el Kunstmuseum Den Haag (KMDH), la conservadora Madelief Hohé, seleccionó diferentes piezas de la colección del museo con la idea de reforzar la visión, a través de la moda y el comercio textil, de los grandes cambios culturales que sufrió Occidente a lo largo de su historia. Es por ello, que algunas de estas piezas allí presentes se decidieron utilizar como objetivo de investigación para entender de una manera más directa el impacto social que la moda generó dentro de la sociedad de cada época. El Departamento de Conservación y Restauración del KMDH junto con el Instituto Valenciano de Conservación y Restauración e Investigación (IVCR+i), decidieron realizar la siguiente investigación de una chaqueta china importada a Europa a principios del siglo $X X$. Fue precisamente esta investigación la que ayudó a entender aspectos técnicos de su producción, así como los procesos de degradación propios de este tipo de objetos.

\section{Contexto social}

Con el final del periodo Qing en 1911 (también conocido como Dinastía Ch'ing), tras aproximadamente cuatros siglos de reinado, mercados externos como el de Occidente, encontraron una grandísima oportunidad para importar objetos chinos cotidianos que fueron transformados bajo un nuevo prisma creativo occidental (Chan 2017: 212). Esta nueva fuente de inspiración, en combinación con los nuevos hallazgos de la arqueología (Turrel 2014: 154), trajo a Occidente una idea idílica de apropiacionismo que influyó tanto en la sociedad como en la cultura de la época (Heroldová 2016: 49).

La forma de vestir también fue modificada, encontrando a través de la figura de los diseñadores más importantes de la época como Jeanne Lanvin, Callot Soeurs o Paul Poiret entre otros, diseños vanguardistas que se nutrían de las fuentes creativas orientales (Heroldová 2017: 35, Martin y Koda 1994: 18). El movimiento del Art Decó de 1920, a través de los movimientos culturales como la egiptomanía, japonismo o chinoiserie, tendieron a agrupar todos estos conceptos erróneamente bajo un mismo prisma cultural, denominado Orientalismo (Chan 2017: 215; Milbank 2010: 569-573). Diseños como el de Paul Poiret condicionaron estas tendencias, como por ejemplo el vestido Pagoda de 1914, el cual conseguiría convertirse en un modelo a seguir de representación de la imagen de Oriente en Occidente (Martin y Koda 1994: 29). Esta fiebre creativa trajo posteriormente colecciones inspiradas a través de los denominados dragon robes o mantones de Manila chinos que eran reinterpretados en los años 20 del siglo pasado a través de figuras tan reconocidas como Callot Soeurs (Heroldová 2016: 49; Martin y Koda 1994: 27). Los años 30 recogieron el testigo iniciado en la década anterior con diseñadores como Agnés Drecoll, el cual reinterpretó chaquetas bolero, inspiradas en vestidos de corte Manchu de finales del periodo Qing (Martin y Koda 1994: 29). La colección del $\mathrm{KMDH}$, conserva diferentes objetos textiles de este periodo tan singular, como el vestido en crepe de seda de color negro bordado con flores y mariposas (número de inventario $\mathrm{CMC} 727 \mathrm{AB}$ ), el cual refleja una inspiración directa de las faldas de mujer de la corte Qing denominadas mamianqun ${ }^{[1]}$ construidas en forma de paneles perpendiculares y bordadas con motivos vegetales y animales. El uso de este tipo de prendas se volvería muy popular entre las casas de moda parisinas como Babani, donde su característica construcción se reconocería a través del tipo de tejidos empleados, así como del trabajo de bordado inspirado en Oriente (Galliera 2007: 264) ${ }^{[2]}$.

La década de los 40 trajo consigo nuevas creaciones que se convirtieron en símbolos sociales idílicos de reinterpretación de China en Occidente (Martin y Koda 1994: 18). Este fue el caso, por ejemplo, del vestido Qipao (también conocido como cheongsam), que resultó ser muy popular para mujeres emancipadas, así como un atuendo convencional para actrices orientales en películas americanas de finales de los años 30 (Chan 2017: 226; Martin y Koda 1994: 9, 19).

Otras veces, sin embargo, en lugar de reinterpretar o crear a través de una perspectiva idílica de Oriente, los objetos originales eran transformados siguiendo el gusto occidental (Heroldová 2016: 49). Por ejemplo, los zapatos característicos utilizados durante el reinado Qing por las mujeres, también conocidos como lotus, fueron reutilizados como soportes de perfumes y maquillajes en Occidente (Chan 2017: 214). Esta idea de posesión relacionada con la belleza eterna y efímera del pasado chino, fue reflejo de propaganda de revistas de la época como Vogue en Francia, enfatizando la idea de adquirir dichos objetos dado su carácter exótico (Chan 2017: 214215). La importación de este tipo de objetos trajo como consecuencia la modificación de trajes oficiales de corte como por ejemplo los denominados anteriormente dragon robes o jifus, los cuales se transformaban en atuendos informales en forma de abrigos o vestidos (Bloomfield 2020: 3; Heroldová 2017: 34-35; Vainker 2004: 170). De hecho, figuras relevantes como Emile Flöge ${ }^{[3]}$ o el actor americano Cliffton Webb fueron retratados vistiendo jifus de manera informal en varias ocasiones (Tretter y Weinhäupl 2016: 49) ${ }^{[4]}$.

\section{Los vestidos jifu o dragon robe}

La importación de estos objetos en mercados europeos y americanos hizo que numerosos museos nacionales como locales conserven en la actualidad muchos de ellos. En España, colecciones como la del Centro de Documentación y Museo Textil en Terrassa (CDMT) conserva dos figurines de finales del XIX que representan los atuendos característicos tanto del emperador como de la emperadora de la dinastía Qing (número de inventario 12316 y 12317). Adquiridos 
posiblemente en alguna de las Exposiciones Universales Europeas celebradas a finales de siglo por el coleccionista Lluís Tolosa, ambos son un testigo directo del modo de vestir, así como del tipo de indumentaria de la clase social alta de la época, conservando todos sus elementos originales sin modificar (Ubach 214: 37).

Desafortunadamente, a diferencia de los ejemplares del CDMT, elvestido deesta investigación (número de inventario K-222-1969) presenta grandes modificaciones de su diseño original siendo un reflejo de importación y modificación al gusto occidental de principios del siglo XX. Diseñado para los hombres más allegados al emperador, fue denominado como jifu o dragon robe. Este tipo de atuendos seguía una jerarquía social muy estricta, definida a través de edictos oficiales como el publicado en la enciclopedia Huangchao liqi tushi de 1796, donde se establecían las normas oficiales de vestir para los miembros de la corte, así como también para la familia del emperador más cercana (Heroldová 2017: 31; Heroldová 2016: 51). Su diseño resultó ser muy sencillo, ya que se basaba en una distribución en forma de A con mangas largas y estrechas que finalizaban en forma de puños de pezuña de caballo. La parte de los hombros no presentaba ningún tipo de costura, siendo únicamente visible aquella ubicada tanto en el frente de la prenda como en la trasera de la misma, ambas camufladas bajo los ricos trabajos de bordado. Las extensiones utilizadas tanto en los laterales, así como en los puños o el cuello a través de la adición de tejidos acanalados de color negro o azul oscuro, completaban el diseño de estas prendas. Por último, los vestidos eran forrados con damascos de seda en combinación de trabillas y bolas de latón que ayudaban al portador a cerrar el uniforme (Heroldová 2016: 53; Sher Ali Khan 2010: 1). El tipo de material empleado en su confección también estaba condicionado por el momento de producción, ya que para periodos de calor se empleaban tejidos más ligeros como, por ejemplo, los conseguidos a través de la técnica denominada kesi. Este tipo de tejido hacía referencia a la técnica de confección, ya que combinaba técnicas de tapiz junto con confección en telar. Para periodos con temperaturas más gélidas, se prefería el uso de tejidos con mayor cuerpo y densidad ya que prevenían del frío. Los trabajos de bordado, así como el color del material seleccionado, estaban igualmente condicionados según el estatus social del portador (Lin y Duarte 2017: 55; Han y Quye 2018: 49). La jerarquía de sexos también estaba definida ya que, por ejemplo, los diseños de las prendas para las mujeres estipulaban la ausencia de las características trabillas y bolas de latón presentes en las prendas masculinas (Lin 2013). Desafortunadamente, el vestido que centra nuestro estudio conserva muy poco del diseño original en la actualidad, reflejando un estilo característico de finales de los años 20 que poco tiene que ver con su función inicial [Figura 1]. Tras consultar con profesionales especializados en este tipo de colecciones como la conservadora Jackie Yoong del Asian Civilizations in Peranakan Museum (Singapur), se pudo datar el diseño original a finales del siglo XIX, siendo el color rojo empleado como soporte, característico de la China tradicional, el cual es asociado con la buena suerte y la fortuna. Esta gama cromática era una reminiscencia directa de los habitantes Manchurians, que únicamente vestían prendas de color rojo $^{[5]}$ (Lin y Duarte 2017: 59; Sher Ali Khan 2010: 10).

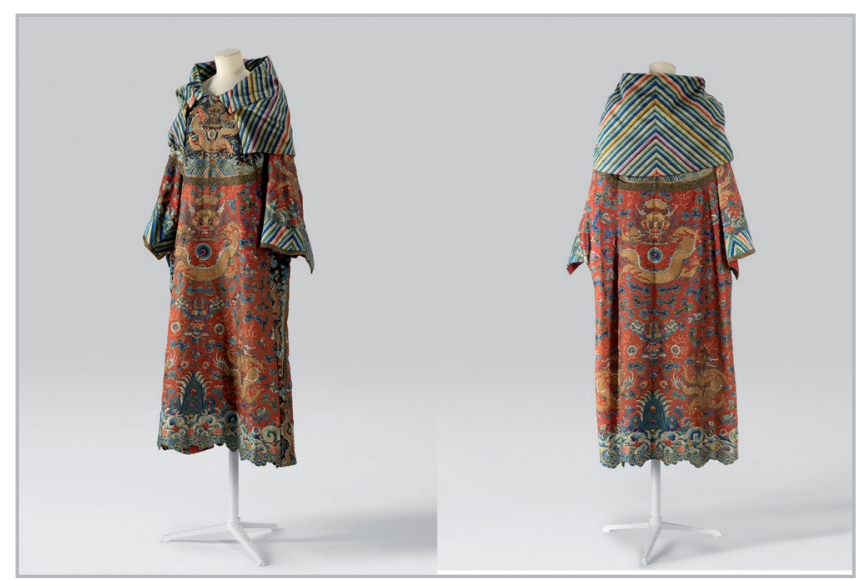

Figura 1.- Jifu modificado en forma de abrigo de noche de finales de los años 20 del siglo pasado. Colección $\mathrm{KMDH}$, fotografía Adriaan van Dam.

Observando otras colecciones museísticas o casas de subastas, se pudieron encontrar ejemplos similares de modificación de diseño durante esta investigación. Este es el caso por ejemplo de la chaqueta Chanel conservada en el Metropolitan Museum de Nueva York (MET), cuyo tejido original corresponde a una prenda jifu ${ }^{[6]}$, o la transformación en forma de vestido de noche vendido en el año 2010 por la casa de subastas Kerry Taylor en Londres ${ }^{[7]}$. Igualmente, autores como Helena Heroldová han podido identificar durante su investigación en el Museo Nacional de Náprstek (MNN) varios vestidos transformados siguiendo esta tendencia de transformación (Heroldová 2017).

\section{Técnica de ejecución}

La primera fase de esta investigación correspondió al estudio pormenorizado de la técnica de ejecución tanto del bordado como del forro, ambos característicos del estilo de este tipo de prendas.

\section{-Bordado}

Para el estudio de los motivos bordados presentes en el jifu de la presente investigación, autores tan importantes del siglo XX como Schuyler, Cammann, John E. Vollmer, Gary Dickinson, Linda Wrigglesworth, Alan Priest, Helena Heroldová o Young Yang Chung (Yang Chung 1976) junto con la ayuda profesional de Jackie Yoong conservadora del Asian Civilizations in Peranakan Museum (Singapur), han servido para interpretar los motivos iconográficos presentes así como entender su significado y su contexto histórico-social en su momento de producción (Heroldová 2017: 29-40; Heroldová 2016: 49-72). 
Durante la dinastía Qing existieron diferentes casas de bordado especializadas según el tipo de indumentaria y clase social. La más importante de todas ellas fue la denominada xiuzhuang ye especializada en la confección de bordados reales (Silberstein 2020:149). Varios fueron los centros de producción principales de estos bordados como Nanking, Hangshou, Suzhou y Guangdong, siendo Nanjin y Suzhou los que mayor reconocimiento obtuvieron debido a la calidad de los hilos dorados empleados (Silberstein 2020: 150). En cuanto a los centros de tinción y producción textil, destacaron Jiangnan (próximo al valle inferior del rio Yangtze) y Beijing, siendo principalmente el algodón y la seda los materiales más utilizados (Han y Quye 2018: 51).

La confección de los bordados podía durar hasta treinta meses, de ahí su exquisitez y finura final. Para ello, el diseño era iniciado en Nanking donde se perfilaban los motivos a representar. A continuación, la tela previamente diseñada era enviada a bordar por varios profesionales especializados en diferentes técnicas de bordado $y$, finalmente, todo este trabajo era estudiado en Pekín a través del consejo de ministros o Board of Rites, donde se dictaminaba la resolución final previa a su comercialización (Sher Ali Khan 2010: 2). Este estricto proceso de producción, condicionado en función del portador de las prendas, seguía edictos oficiales dictados durante la dinastía Qing. No sería hasta finales del siglo XIX cuando el Régimen permitió una mayor libertad de representación de los motivos decorativos presentes en las prendas oficiales (Heroldová 2016: 56).

El estudio de los bordados presentes en esta chaqueta, identificó una iconografía característica tanto del Taoísmo como del Budismo. En el primer grupo iconográfico, el referente al Taoísmo, se determinó por la representación de los ocho inmortales ${ }^{[8]}$. Su simbología era identificada a través de elementos como el abanico portado por Chungli Ch'üan, la espada de Lü Tung-pin, la vara de bambú de Chang Kuo, las castañuelas de Ts'ao Kuo-chiu, la flauta de Han Hsiang Tzŭ, la doble calabaza de Li T'ieh-kuai, la Canasta de Lan Ts'ai-ho y el Loto de Ho Hsien-ku. En cuanto al grupo de índole budista, su iconografía se centraba en otros ocho motivos tales como el dosel, la concha, el jarrón, la bandera real, la rueda de la ley, un par de peces confrontados, la flor de loto y un nudo sin fin (Sher Ali Khan 2010: 6) ${ }^{[9]}$. De todos ellos, en el vestido se identificaron el abanico, la flor de loto, la caña de bambú, las castañuelas, la canasta de flores, la pareja de peces o las figuras del dosel [Figura 2].

En cuanto a los motivos más figurativos como el dragón, éste centró gran parte de protagonismo del abrigo, observándose, al menos, ocho dragones en la prenda: tres localizados en el frente, tres en la trasera y dos en los hombros, sin contar la presencia de un último dragón escondido bajo la apertura de la solapa del abrigo en el frente. Este noveno dragón, junto con la representación de las cinco garras, justificó el estatus social del portador de la prenda, ya que en su origen, la representación de nueve dragones con la suma de sus garras, estaba reservada para personas de la corte que trabajaban en el entorno del emperador (Lin y Duarte 2017: 55; Heroldová 2016: 53; Heroldová 2017: 32). Todos ellos presentaban posturas contorneadas y actitud juguetona, apareciendo rodeados de la denominada perla en llamas, la cual se convirtió en uno de los símbolos más comunes en China tanto en tejidos como en artes decorativas (Wilson 1990: 286). Bajo el dragón central de mayor tamaño, se observa la denominada bandera de la victoria, rodeada de cintas de seda de color azul, también conocidas como ruyis. Este motivo central, siempre representado bajo la figura del dragón, podía presentar diferentes formas como sombrillas
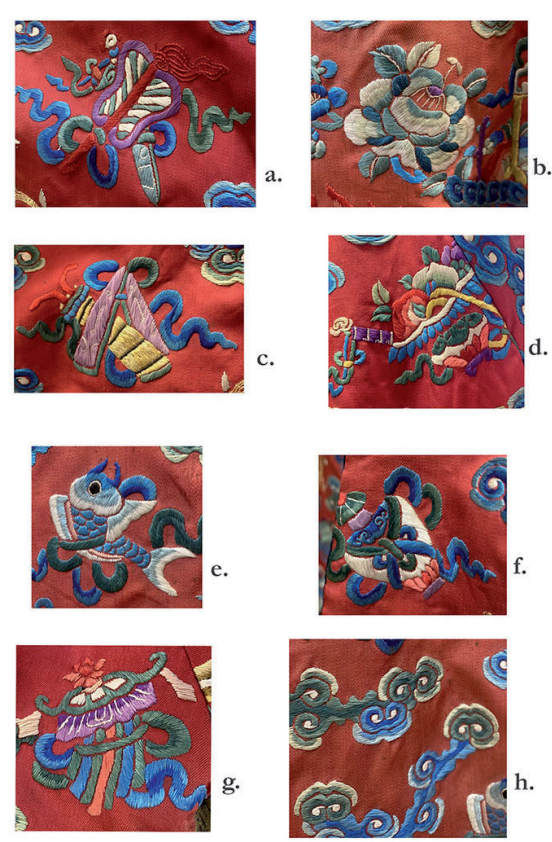
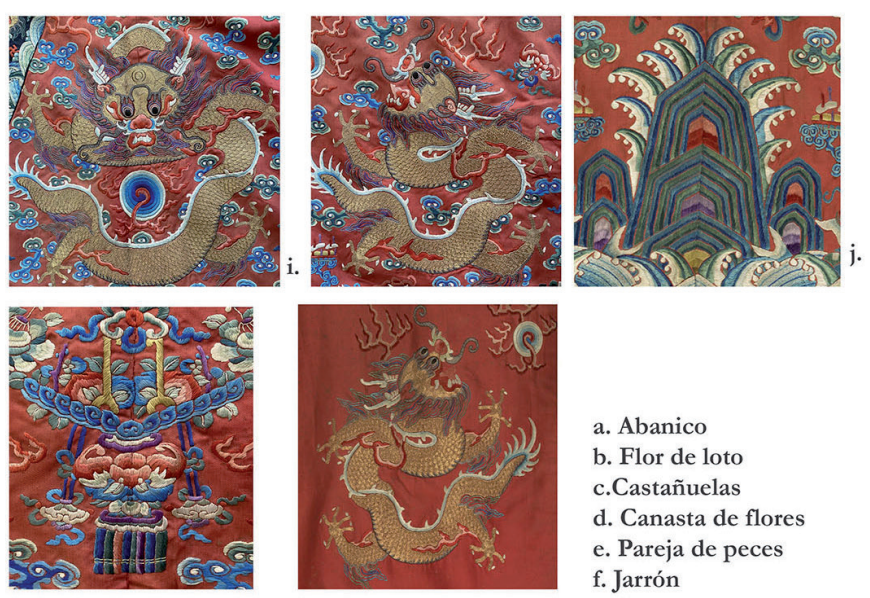

k. 1.

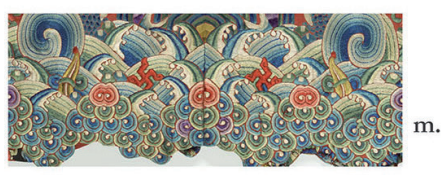

a. Abanico

b. Flor de loto

c.Castañuelas

d. Canasta de flores

e. Pareja de peces

f. Jarrón

g. Dosel

h. Nubes

i. Dragones de 5 garras

j. Montaña Sagrada

k. Bandera de la victoria

con ruyis y murciélago

1. Dragón escondido

m. Aguas turbulentas

Figura 2.- Representación iconográfica de los motivos presentes en el abrigo. Fuente: KMDH 
chinas o cestas de flores las cuales eran combinadas con frutas (habitualmente, melocotones) (Heroldová 2016: 56).

Por último, el resto de la superficie textil estaba decorada con formas abstractas como las nubes, la montaña sagrada o las aguas turbulentas. Diferentes autoras como Helena Heroldová han identificado a través del estudio de las formas de las nubes, así como el tamaño de la composición de las aguas turbulentas, una posible fuente de datación de estas chaquetas, ya que tanto su morfología como su diseño evolucionó a través de los siglos (Heroldová 2016: 56; Garret 1999: 34-52).

Los tejidos utilizados como fondo de los bordados se identificaron como sargas 2:1 S de color rojo y negro. En cuanto a las técnicas de ejecución empleadas para el bordado, estas eran muy variadas, desde la aplicación de punto al pasado o punto treversado para el relleno de grandes superficies empleando hilos de seda sin torsión, hasta el empleo de puntos de costura utilizados todos ellos para la fijación de los hilos metálicos entorchados. Éstos eran fijados al soporte principal a través de hilos de seda sin torsionar, agrupándose en parejas de dos hasta crear las características formas de los dragones. Bajo estos hilos metálicos, se encontraban los denominados canutillos de seda, elaborados todos ellos con sedas de color marrón de dos cabos y una torsión en Z. Por último, la composición estaba finalizada a través de las denominadas puntadas de contorno que ayudaban a cerrar la composición de los motivos figurativos. El estudio meticuloso de estos bordados permitió identificar una ligera línea de color blanca relacionada con su producción, ya que autores como Dusenberry, describen que, previa a la confección de estos bordados, los motivos eran dibujados sobre el tejido con polvo de ostras mezclado con agua tibia y aplicado sobre la superficie del tejido con pincel (Dusenberry 2004: 181). A los profesionales que desarrollaban esta tipología de confección tan meticulosa se les denominó white-chalk drawer en la literatura anglosajona o hua baifen en chino (Silberstein 2020: 163) [Figura 3].

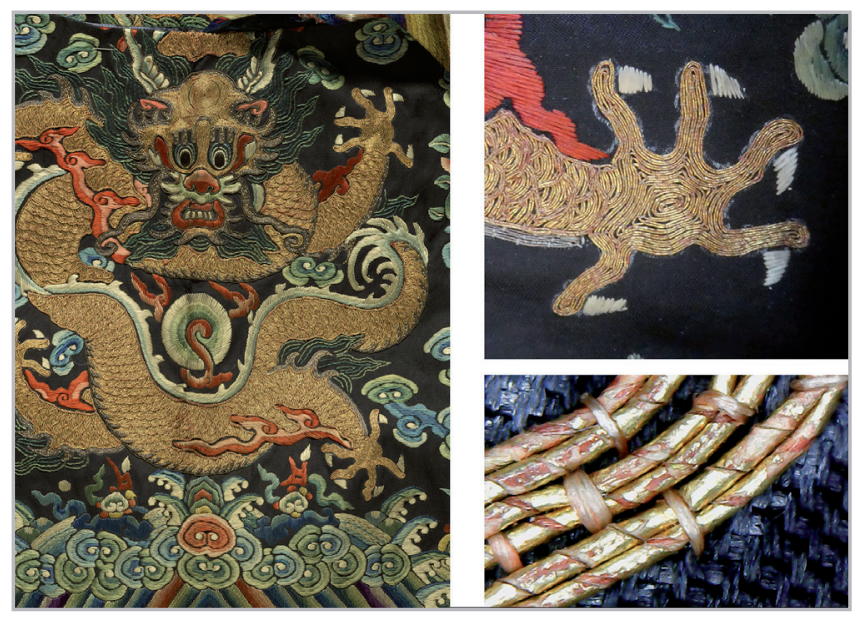

Figura 3.- Registro de la denominada puntada de sutura sobre fondo azul y presencia de la característica línea de color blanco procedente del polvo de ostras alrededor de la garra del dragón. Fuente: KMDH.
- Forro

El estudio del forro también resultó ser muy importante para la investigación, ya que el forro actual era reflejo del momento en el que la prenda original se había transformado en un diseño de los años 20, mientras que los forros originales presentes en este tipo de prendas jifu, son damascos de seda con un complejo diseño de confección. Estas diferencias se observaron a través del estudio de diferentes prendas de la colección del $\mathrm{KMDH}$ (véase por ejemplo K-221-1985 o K-393-1981) así como la consulta de otras colecciones como la del Asian Civilizations in Peranakan Museum (Singapur) donde se conservan un gran número de prendas jifu sin modificar $^{[10]}$.

El nuevo forro añadido al abrigo, fue realizado en damasco de seda decorado con formas florales en color azul y negro, siguiendo un rapport ${ }^{[11]}$ compuesto por dos tipos de flores y diversas hojas. Este tipo de iconografía se inspiraba directamente de los tejidos franceses, con especial atención a las sedas lionesas. Autores como BernardTassinari, publicaron en el año 2012 "La Soie á Lyon. De la Grance Fabrique aux Textiles du XXe Siécle" donde se pudo registrar la evolución completa de la industria textil sedera de Lyon desde sus inicios en el siglo XVIII. Es precisamente en una de las páginas de esta publicación, donde el documentalista Julen Morrás Azpiazu identificó patrones de tejidos similares al aquí estudiado $^{[12]}$, más concretamente al diseño confeccionado por el artista francés J.B. Loir en 1928, extraído de "Cours de théorie du tissage et des étoffes de soie" (Bouzard 1997: 100). Otros países como Inglaterra continuaron esta tradición textil, ofreciendo a través del estudio de tejidos conservados en el Victoria and Albert de Londres (V\&A), tejidos similares producidos por fabricantes como Warner and Sons ${ }^{[13]}$. El uso de este tipo de seda fue muy habitual durante los primeros años del siglo XX como se observa en las colecciones del $M E T^{[14]}$ y el $K M D H^{[15]}$.

\section{Estado de conservación}

En términos generales, el estudio del tejido base de color rojo utilizado para la aplicación de los bordados, presentaba un buen estado de conservación. Sin embargo, se observaban diferentes pliegues tanto en las zonas de las mangas como en las partes centrales de los motivos figurativos de los dragones. Estos pliegues -muy posiblemente creados por un almacenamiento inadecuado previo a su entrada en la colección del museo-, condicionaban su correcta interpretación. Igualmente, se pudieron registrar manchas de color marrón de tipo foxing alrededor de las costuras que podían estar relacionadas con un posible ataque de microorganismos previo a su entrada en la colección. Esta suposición no pudo confirmarse hasta el tratamiento de conservación-restauración posterior donde el forro fue eliminado y posibilitó su observación detallada [Figura 9].

Evidentemente, uno de los mayores daños que la prenda había sufrido corresponde al momento de su modificación 
en los años 20, donde el objeto sufrió desde pérdidas de material hasta mutilaciones. Es por ello que, se decidió realizar un estudio de patronaje detallado para registrar los daños sufridos por las modificaciones llevadas a cabo. Esta información pudo extraerse gracias al ya mencionado estudio comparativo de prendas jifu no modificadas, como por ejemplo el K-103-1973 de la colección del KMDH. Los primeros resultados obtenidos fueron asombrosos, ya que reflejaban un aspecto cuidadoso a la hora de su modificación, ofreciendo un gran respeto hacia el material original. A rasgos generales, las mangas fueron recortadas y transformadas en mangas francesas abiertas, reubicando sus extremidades compuestas por un tejido acanalado de color negro, en forma de solapa que cubría tanto la parte central del abrigo como la parte superior de la espalda. Parte de estas extremidades también fueron reutilizadas para ensanchar los laterales del abrigo, creando de esta manera un juego cromático a la hora de ser vestido. En cuanto al cuello alto, constituido por las denominadas líneas diagonales ascendentes, estas fueron las que mayores pérdidas sufrieron respecto al diseño original, ya que, en su aspecto primitivo, recorrían la parte del bajo del vestido, habiéndose conservado únicamente un tercio del original. Igualmente, se pudo observar su transformación a través de los nuevos cortes y costuras que fueron añadidos al diseño original, ofreciendo un nuevo tipo de unión entre los tejidos [Figura 4].

Durante el estudio detallado de los cambios sufridos, se pudo documentar la existencia de agujeros originales correspondientes a la ubicación de trabillas de cierre de la prenda. Este hecho reflejaría que, en su origen, el vestido fue llevado por un hombre y no por una mujer como ahora se presentaba (Heroldová 2017: 34).

El trabajo de bordado estaba en bastante buen estado de conservación, ya que, prendas similares estudiadas en otras colecciones, presentaban parte del bordado desprendido o perdido de su ubicación original debido a la degradación característica de los hilos de fijación de seda empleados sobre los hilos metálicos ${ }^{[16]}$. Esta característica común de degradación presente en otras prendas jifu, no se observaba en la chaqueta de estudio, puesto que presentaba estabilidad y buena resistencia. Sin embargo, el estudio detallado de las láminas metálicas con microscopio estereoscópico (Carl Zeiss $^{\mathrm{TM}}$ ) registró, pérdidas de material sobre las láminas de oro, zonas despegadas del soporte de papel original empleado para la adhesión de estas láminas, así como ennegrecimiento generalizado en los hilos metálicos de color gris, muy posiblemente relacionados con procesos de corrosión. Todas estas características estarían relacionadas con los procesos de producción de estos hilos, así como con las condiciones a las que la prenda fue sometida previo a su entrada a la colección del museo, ya que durante la dinastía Qing, el empleo de estos hilos metálicos se caracterizó por el uso de aleaciones susceptibles a la corrosión en presencia de altos niveles de humedad relativa (Tímár-Balázsy y Eastop 2008: 137).

Continuando con el estudio de daños de la prenda, el forro era sin duda el que mayor atención suscitaba debido a su

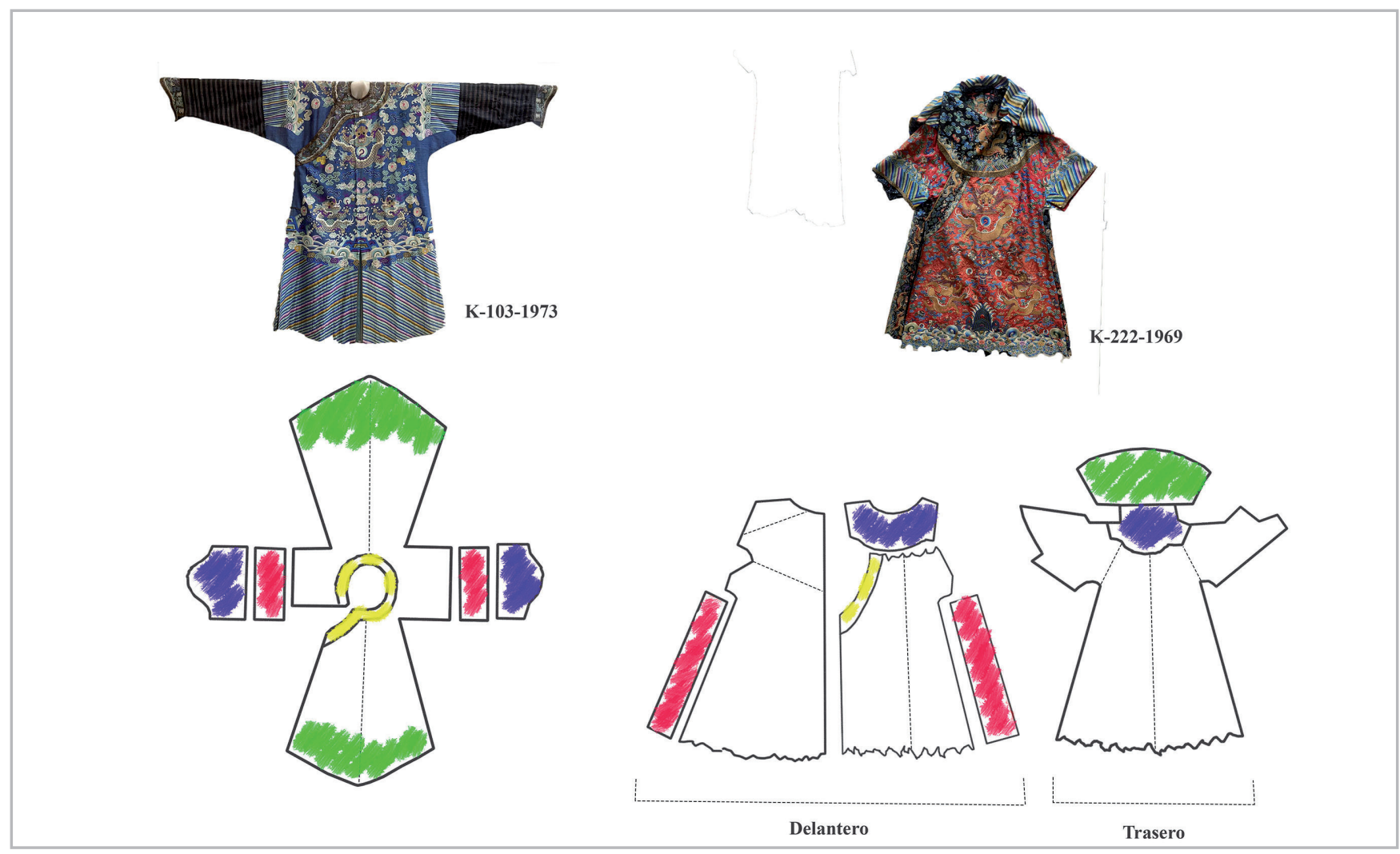

Figura 4.- Esquema comparativo de un jifu sin modificación (K-103-1973) con respecto al abrigo modificado (K-222-1969). Los colores representan la ubicación original de los fragmentos con respecto a un jifu sin modificar. Fuente: KMDH. 
mal estado de conservación. Presentaba una fragilidad extrema, con pérdidas de material que afectaban tanto a las urdimbres como a las tramas, siendo sobre todo estas últimas las que mayor fragilidad mostraban, con roturas características en sentido longitudinal que modificaban el aspecto original de la composición del forro [Figura 5].

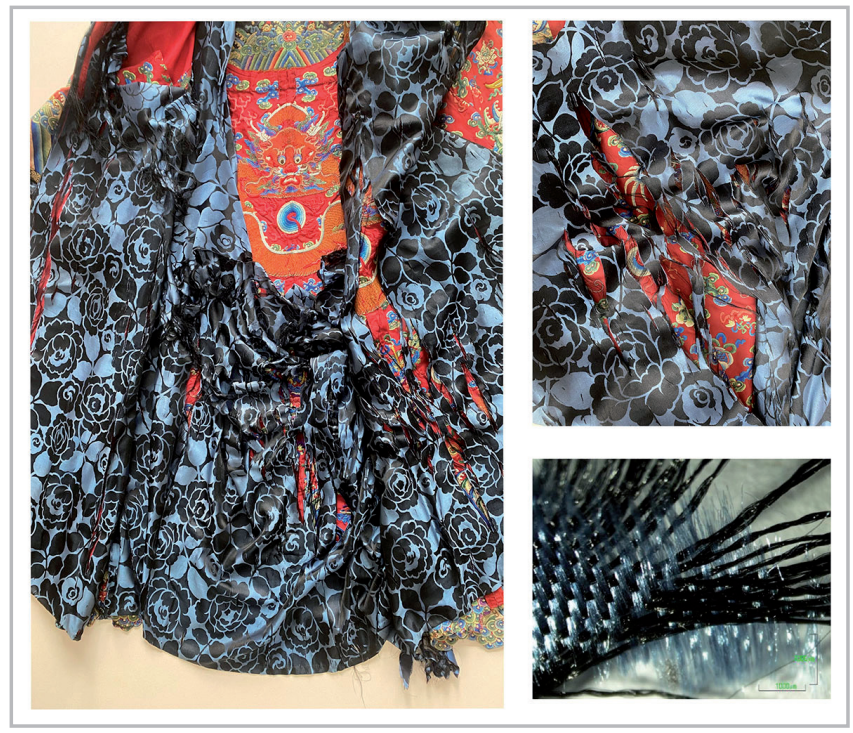

Figura 5.- Estado de conservación del forro antes de su tratamiento y observación bajo microscopio estereoscópico (30x) mostrando la ruptura característica de las tramas de color azul. Fuente: $\mathrm{KMDH}$.

\section{Caracterización de los materiales}

Con la intención de caracterizar los materiales constituyentes del vestido, así como sus causas de alteración, se recogieron cinco muestras para análisis en el laboratorio. Estas muestras pertenecientes al bordado y al forro, se sometieron a diferentes fases de estudio con diversas técnicas de análisis. La primera de ellas correspondió al microscopio estereoscópico Nikon SMZ1000 con el que se realizaron microfotografías con cámara digital acoplada Nikon DS-2Mv (10x-80x aumentos) para identificar el tipo de muestra a estudiar.

A continuación, se procedió al estudio morfológico de las muestras con microscopía óptica, microscopía estereoscópica y microscopía electrónica de barrido. Para ello, se utilizó un microscopio óptico Nikon ECLIPSE $80 \mathrm{i}$ con cámara Nikon DS-Fi1, provisto de luz reflejada y polarizada con iluminación UV (50x-500x aumentos), así como un microscopio electrónico de barrido Hitachi Ltd (SEM) modelo S-3400N en modalidad de electrones retrodispersados (BSE) (50x-2000x aumentos).

Para terminar, el empleo de microscopía electrónica de barrido acoplada con microanálisis por dispersión de energías de rayos $X$ (SEM-EDX) con un equipo Bruker Corporation XFlash ${ }^{\circledR}$ de un voltaje de aceleración 20 kV, sirvió para caracterizar los diferentes componentes presentes tanto en los hilos de bordado así como en las muestras del forro recogidas.

\section{- Estudio de los hilos metálicos}

Para el análisis de los hilos metálicos, fue necesaria la extracción de cuatro muestras. Empezando por la identificación de los componentes de degradación, su estudio a través de microscopía estereoscópica sirvió para identificar la presencia de láminas doradas con alteración cromática en forma de pátina oscura y craqueladuras, así como la presencia de un estrato rígido de color ocre con trazas de color rojo en el que se asentaba la lámina metálica. Bajo ésta -y gracias a los resultados obtenidos por microscopía óptica-, se pudo identificar un alma de color blanco compuesto por cinco fibras de seda que componían un hilo final torsionado en S [Figura 6 a-b]. Sobre ésta, la lámina metálica se enrolla en espiral siguiendo una torsión Z característica de estos hilos metálicos. Las observaciones a través de SEM de las lagunas presentes bajo la lámina metálica, confirmaron la presencia de diferentes estratos propios de los procesos de elaboración de los hilos metálicos [Figura 7-a].

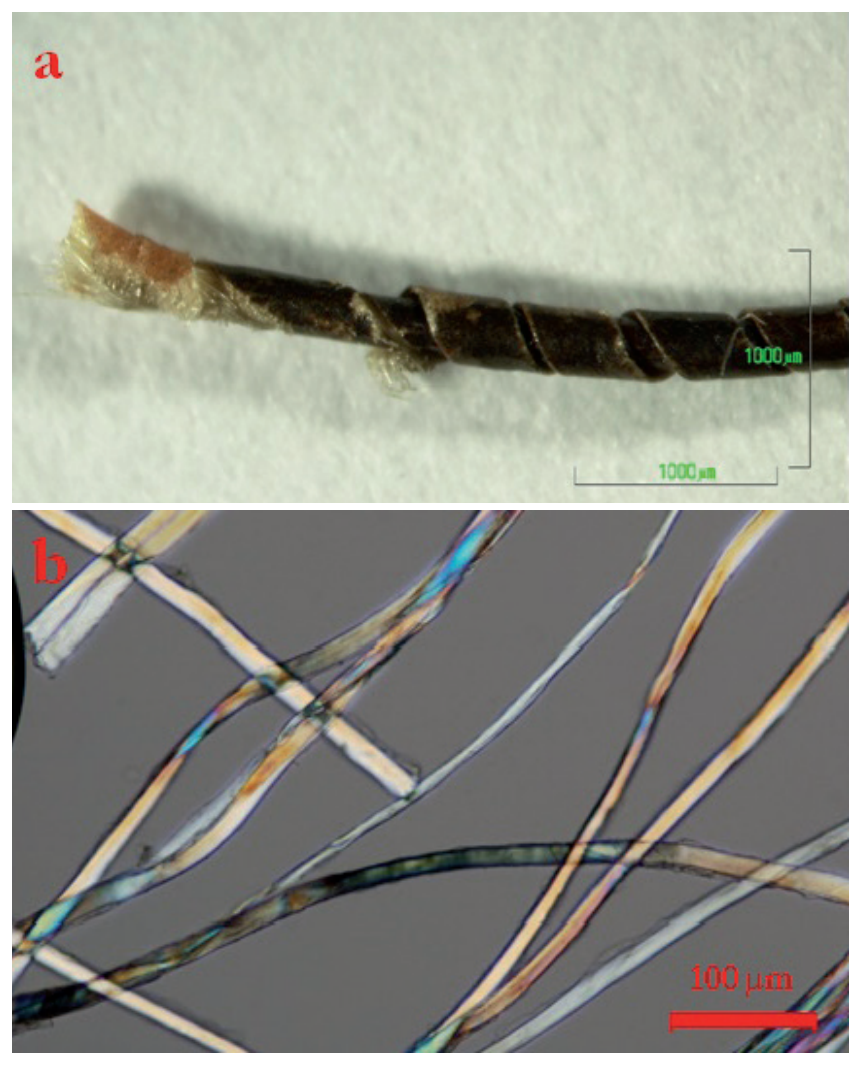

Figura 6.- . (a) Imagen de microscopía estereoscópica de la muestra de entorchado dorado con una ligera pátina negra en superficie producida por los fenómenos de degradación de la plata, 30x. (b) Imagen de microscopía óptica de las fibras de seda en sección longitudinal, 200x. Fuente: IVCR+i.

Los resultados obtenidos a través de microanálisis EDX, confirmaron la presencia tanto de oro como de plata, además de elementos de degradación característicos como el cloro y el azufre, causantes del ennegrecimiento característico de la plata [Figura 6-a y 7-b]. El uso de aleaciones metálicas para la elaboración de estos hilos 
fue una práctica común durante el periodo Qing, lo cual condicionó tanto el color como su estado de conservación. Varios autores hacen referencia al uso de aleaciones para la confección de hilos metálicos como por ejemplo el oro y la plata, el cobre, el latón o el estaño (Nord y Tronner 2000: 274-279).

El análisis de los materiales observados bajo las lagunas producidas por la pérdida de material permitió identificar la presencia de un material de naturaleza orgánica elaborado con fibras de celulosa, muy probablemente papel [Figura 7-a]. Sobre este estrato, se identificaron además elementos químicos como silicio, aluminio, hierro, potasio y calcio [Figura 7-b], todos ellos asociados a compuestos como aluminosilicatos de hierro, o arcilla roja. La identificación de estos elementos justificó su lugar de procedencia, ya que su empleo fue únicamente registrado en China durante este periodo. Su elaboración seguía un proceso meticuloso en el que las láminas metálicas eran adheridas a soportes celulósicos que eran previamente mordentados con una arcilla de color rojo, también identificada como hematita $\left(\mathrm{Fe}_{2} \mathrm{O}_{3}\right)$ o bol de color rojo (Han 2016: 257; Hwei-Fe'n 2015: 250). Como proceso final, las láminas se recortaban en finas cintas que eran enrolladas alrededor de un núcleo de seda $\mathrm{Hu}$
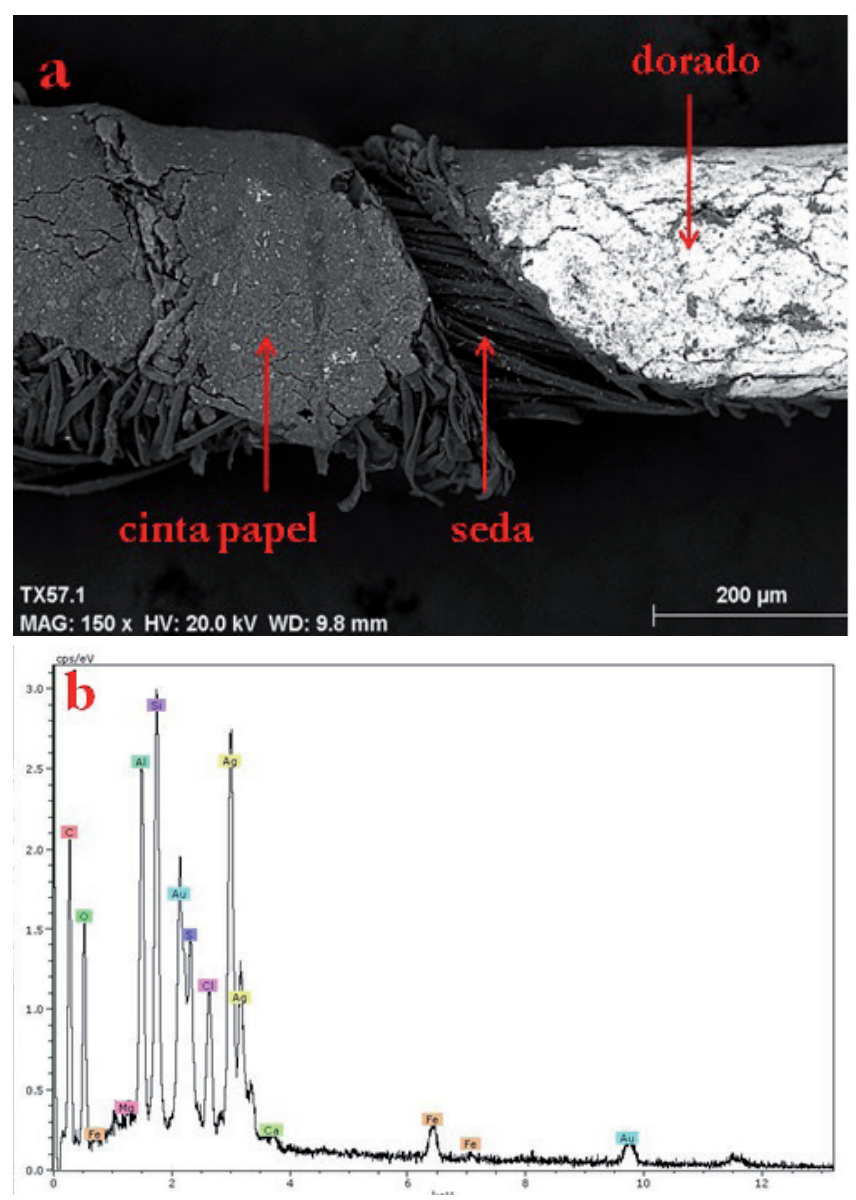

Figura 7.- (a) Imagen SEM en modalidad electrones retrodispersados BSE de la sección longitudinal del entorchado. (b) Microanálisis EDX del dorado. Fuente: IVCR+i.
2016: 135). Esta tipología de hilos se ha documentado en la China imperial, sobre todo a partir de la dinastía Tang (618-907 d.C.) continuando su producción a lo largo de los siglos posteriores (Martin y Koda 1994: 20; Garrett 1990: 54). Pocos estudios han identificado la presencia de este tipo de material hasta la fecha, mereciendo destacar las investigaciones de Jing Han en varios ejemplares de la colección del V\&A documentando varios hilos metálicos con características similares a los aquí estudiados (Han 2016: 260), o los realizados por Cheah Hwei-Fe'n sobre objetos similares procedentes de Indonesia y China (Hwei-Fe'n 2015: 250). Estas investigaciones ayudaron a ubicar la procedencia del vestido objeto de estudio en China.

\section{- Estudio del forro}

La segunda parte de los análisis se centró en el estudio de la muestra del forro. Esta, estaba compuesta por una trama de color azul y una urdimbre de color negra. Ambas fibras mostraban una pérdida de resistencia mecánica evidente, siendo la primera de ellas la más afectada. La observación a través de microscopía óptica y electrónica, identificó seda como componente principal para ambas fibras [Figura 8 a-b]. Para entender el mecanismo de degradación que habían experimentado, las dos fibras fueron sometidas a microanálisis EDX, identificando en las fibras azules altas concentraciones de silicio, estaño y fósforo, mientras que para las fibras de color negro únicamente se registró estaño [Figura $8 \mathrm{c}-\mathrm{d}$ ]. La identificación de estos elementos químicos indicaría la presencia de seda cargada, producida a través del denominado proceso industrial de fosfosilicato estánico. Este proceso fue una gran innovación tecnológica a finales del siglo XIX de la que la industria textil obtuvo grandes beneficios económicos, a pesar de su gran inestabilidad debida a los fenómenos de hidrólisis y oxidación (Hacke 2009, 4-5). Su ventaja respecto a procesos anteriores consistió en que permitía maximizar su producción hasta un 400\% más en relación a su volumen original (Luxford 2011: 3, Garside, Wyeth y Zhang 2010: 179; Luxford 2009: 12). Patentado en 1897 en Estados Unidos bajo el número 583.725, su proceso de degradación se iniciaba en el mismo momento de su producción. La seda era sometida a diferentes soluciones acuosas como cloruro de estaño, fosfato de sodio y/o silicato de estaño, que generaban un baño excesivamente ácido condicionando el estado final de la seda. Este proceso fue al menos utilizado por la industria textil hasta 1920, patentando, a posteriori, otros tipos de mecanismos como los baños con ácido fosfórico o ácido fórmico, que tampoco mejoraron los procesos previos de fabricación (Montero 2011: 92-93; Horswill y Young 1992: 4).

Como consecuencia las sedas acababan rompiéndose en sentido longitudinal a través de su fibra más debilitada (la trama en la mayoría de las veces), impidiendo que pudieran volver a ser utilizadas. 

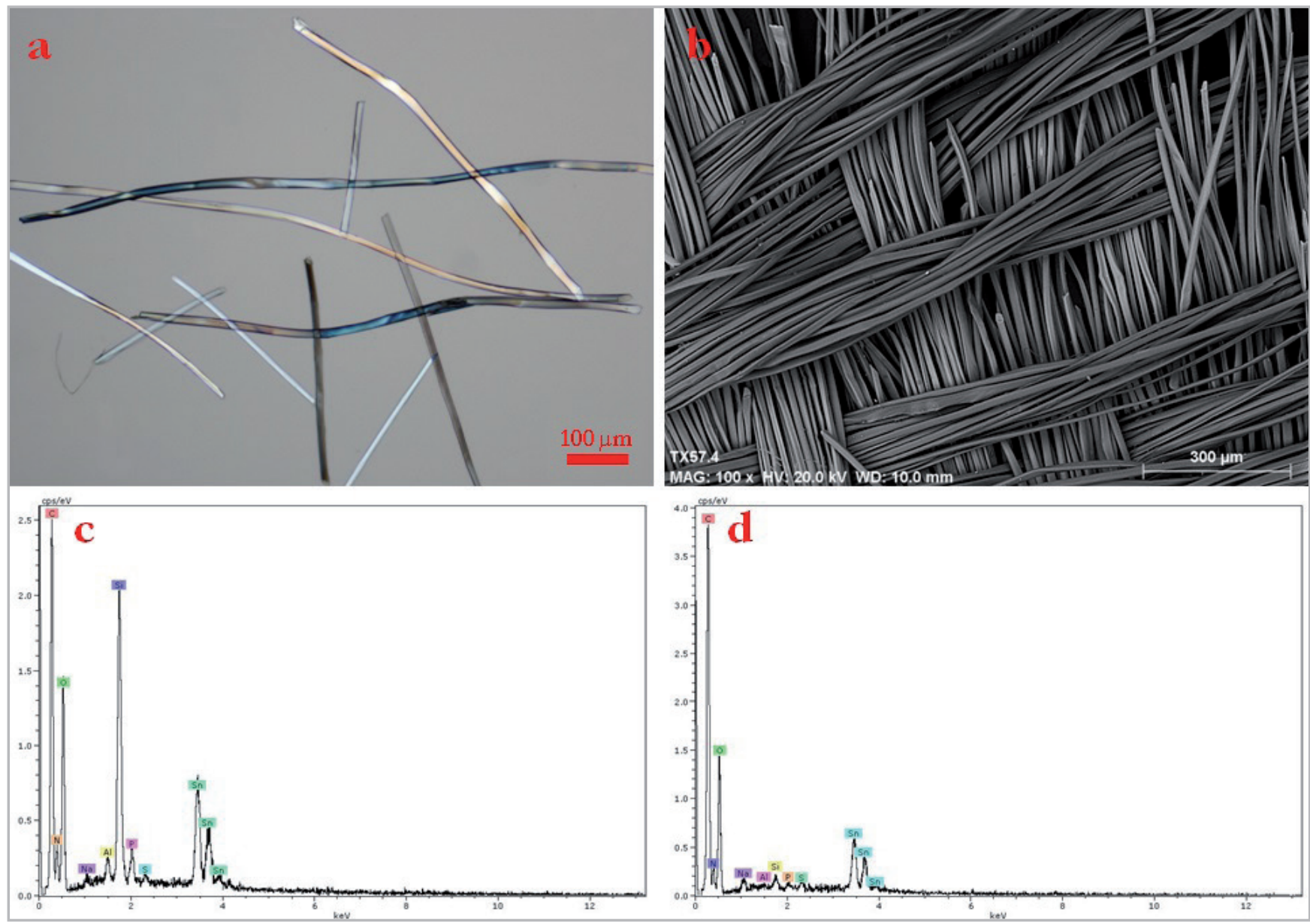

Figura 8.- (a) Imagen de microscopía óptica de las fibras de seda azul en sección longitudinal, 100x. (b) Imagen SEM en modalidad electrones retrodispersados BSE del forro. (c) Microanálisis EDX de las fibras de seda azules. (d) Microanálisis EDX de las fibras de seda negras. Fuente: IVCR+i.

\section{Tratamiento de conservación-restauración}

Tras los análisis, se comenzó con el tratamiento de conservación-restauración. Dado que la transformación del vestido era un reflejo histórico del momento en el que la prenda se trajo a Occidente, se decidió emplear todos los recursos disponibles para conservar el forro interior de la prenda, y consolidar aquellos materiales en peligro de conservación. Así pues, se desestimó desde el principio la posibilidad de reconstruir el diseño original, ya que su actual construcción era un reflejo directo del comercio textil desarrollado durante el siglo XX. Dicha modificación supondría, por lo tanto, la eliminación histórica del momento de importación del objeto a Occidente.

Los análisis de los bordados sirvieron para identificar los problemas de degradación presentes, como por ejemplo las pérdidas de material de las láminas de oro y los productos de corrosión. La estabilización e intervención de los hilos metálicos se dividió en dos fases de trabajo. La primera correspondió a la estabilización de las láminas de oro despegadas del soporte celulósico original. Para ello, se realizaron tests con diferentes tipos de adhesivos bajo microscopio estereoscópico. El primero de los adhesivos testados fue carboximetilcelulosa (CMC) utilizada previamente en otras investigaciones para la fijación de este tipo de hilos metálicos aplicados sobre base orgánica como el cuero (Gill y Boersma 1997: 17). Los resultados obtenidos de dicho proceso no fueron del todo los esperados ya que, además de su compleja aplicación a través de pincel, no terminaban de adherirse de manera uniforme sobre el soporte celulósico. Es por ello que se testaron otro tipo de adhesivos como los acrílicos, puesto que su aplicación en estado líquido permitía una mejor manipulación que el estado gelatinoso de la CMC. De todos ellos, se decidió emplear Lascaux $498^{\circledR}$, ya que su empleo se encontraba referenciado en soportes similares como las faldas chinas Mamianqun, donde los hilos metálicos fueron fijados a través del empleo de este adhesivo (Carbone 2016: 11) ${ }^{[17]}$. Con mucho cuidado y evitando depositar adhesivo en la superficie que pudiera modificar su aspecto original, las láminas metálicas fueron consolidadas devolviéndoles, así, su aspecto original. En cuanto a la eliminación de los productos de corrosión presentes en los hilos metálicos, estos no resultaron ser una prioridad para la buena conservación de la prenda, ya que su eliminación podría afectar al soporte original y contribuir a la pérdida de material de la lámina metálica (Tímár-Balázsy y Eastop 2008: 242-247) ${ }^{[18]}$. 
El soporte original de ligamento sarga de color rojo que presentaba diferentes pliegues debido a un almacenamiento inadecuado, fue igualmente intervenido. Para ello, se empleó primero un tratamiento local con Goretex ${ }^{\circledR}$, papel secante humedecido y peso controlado. Este proceso permitió controlarlaintroducción dehumedad en el soporte original y proceder a la eliminación de las deformaciones de manera controlada. A continuación, el empleo de vapor frío con alfileres entomológicos y cristales, ayudó a paliar dichas deformaciones. Tras la eliminación del forro, se pudo observar la presencia de grandes superficies de engrudo natural, causantes de generar las manchas previamente identificadas en superficie, probablemente producidas por un ataque biológico antiguo. Estas manchas se decidieron mantener como reflejo histórico de producción ya que gracias a la información compartida por la conservadora y restauradora textil Miki Komatsu en piezas similares conservadas en la colección del Asian Civilizations in Peranakan Museum (Singapur), el departamento pudo identificar que dichas prendas eran originalmente preparadas con engrudos naturales antes de su fijación mediante costura. La eliminación de estas manchas hubiera supuesto perder este rastro de originalidad y, teniendo en cuenta que el ataque biológico no estaba activo, se decidió no proceder a su eliminación [Figura 9] (Morgan 2016:11).

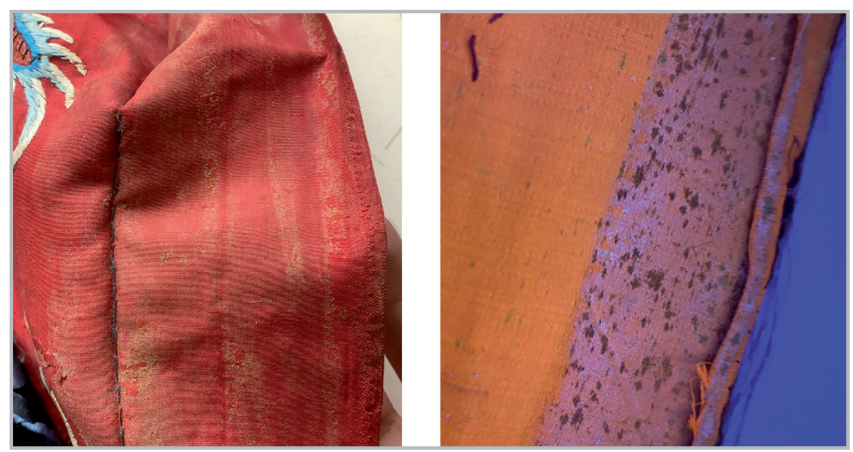

Figura 9.- Observación con luz natural (izq.) la presencia de restos de adhesivo empleado para la unión de las costuras y (dch.) imagen con UV de la misma zona con restos de actividad biológica. Fuente: $K M D H$.

En cuanto al forro, el tratamiento de conservación fue diferente. El problema presente en este tipo de sedas cargadas ha sido, a lo largo del siglo XX, uno de los debates más comunes dentro de los museos con colecciones de indumentaria histórica. Son varias las metodologías de conservación llevadas a cabo a lo largo de estos años, desde la sustitución del original hasta la consolidación del mismo a través de técnicas invasivas como el empleo de adhesivos de base acrílica u otros, menos invasivos que estos, como el encapsulado de los materiales dañados. Ninguno de estos tratamientos ofrece en la actualidad una certeza de estabilidad $y$, mientras, los tejidos siguen degradándose con el paso del tiempo de manera irreversible. Sin embargo, dado que el principal objetivo de este proyecto era su exposición, los criterios del $K M D H$ siempre se han regido por salvaguardar los materiales originales junto a los objetos expuestos. Fue por ello, por lo que la decisión final se basó en la conservación del forro como reflejo histórico de su modificación.

El primer paso llevado a cabo consistió en el estudio de patronaje del forro. Esta fase ayudó a valorar, por una parte, el número de pérdidas presentes en el damasco de seda y, por otra, a identificar el tipo de trabajo llevado a cabo durante la transformación del jifu en un abrigo de finales de los años 20 [Figura 4]. En su totalidad, seis fueron las piezas textiles empleadas para la elaboración del forro, las cuales se sometieron de manera individual, a un proceso de humectación con vapor frío y se alinearon siguiendo la dirección natural de las urdimbres y las tramas. A continuación, se procedió a la tinción de una seda habotai y un tul de celdas ligeramente abiertas que mimetizaban los colores del damasco de seda. Teñidos con tintes sinéticos como Lanaset ${ }^{\circledR}$, estos nuevos textiles sirvieron como soporte de protección, los cuales, a pesar de no poder estabilizar los mecanismos de degradación de la seda, sirvieron como material de encapsulado para evitar pérdidas mayores de material original durante su manipulación. Por último, cada pieza textil que componía el forro, fue unida con hilo de seda de dos cabos Güttermann ${ }^{\circledast}$ y devuelta a su posición original [Figura 10].

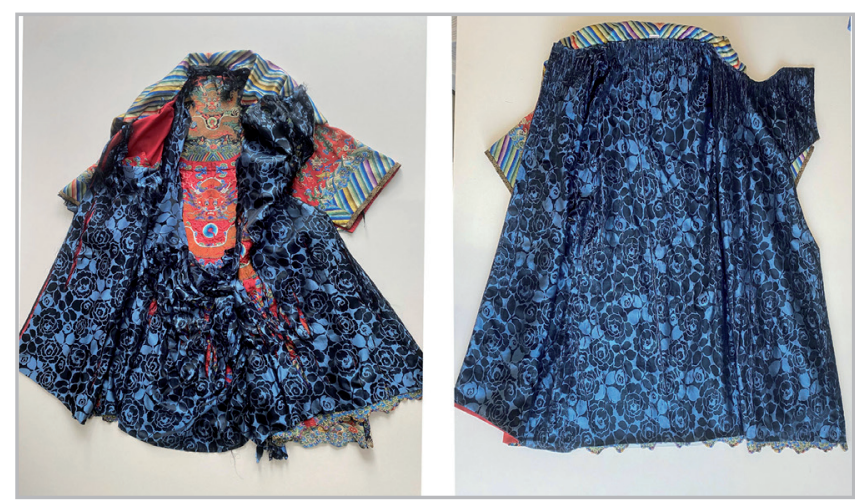

Figura 10.- Imagen del forro del cuello, antes y después de su tratamiento. Fuente: $\mathrm{KMDH}$

\section{Conclusiones}

A través del presente estudio de una chaqueta jifu, se ha podido identificar un momento social que tuvo lugar en Occidente a principios del siglo XX con el éxtasis del denominado movimiento orientalista. La descontextualización de gran parte de estos objetos bajo un prisma occidental fue una práctica habitual durante este momento en el que su asociación fue característica del lujo y el exotismo. Su estudio ha servido para identificar su origen, siendo una prenda masculina transformada en abrigo femenino y traída a Europa tras la caída del imperio Qing en 1911.

Por otra parte, el estudio de las pérdidas y modificaciones 
llevadas a cabo en la prenda junto con los análisis científicos realizados, identificaron los procesos de degradación activos dentro de los hilos metálicos y extrajeron una mayor información de sus procesos de producción, ubicando el abrigo originalmente en China. Por último, el análisis del forro sirvió para entender el grave estado de conservación que presentaba y que reflejaba un tipo de intervención habitual en la industria textil tanto a finales del siglo XIX como principios del siglo $X X$. A pesar de no poder remediar su degradación con los procedimientos actuales y dado su importancia dentro de la pieza de estudio, se procuró conservar el material original a través de un tratamiento de conservaciónrestauración que asegurará su preservación al menos, a corto medio plazo.

\section{Agradecimientos}

Los autores agradecen a todas aquellas personas involucradas en la investigación a través de su consejo y apoyo profesional: Doede Hardemann, Madelief Hohé, Jackie Yoong, Miki Komatsu, Julen Morrás Azpiazu y Gemma Contreras Zamorano.

\section{Notas}

[1] Obsérvese por ejemplo la falda de finales del XIX de la colección del Cooper Hewitt museum (número de inventario 18563133) https://collection.cooperhewitt.org/ objects/18563133/ [Acceso 05/02/2021].

[2] Obsérvese la chaqueta en forma de falda china plisada conservada en la colección del Museo Galliera de París (número de inventario: 1964.20.119). https://www.parismuseescollections. paris.fr/es/node/195324 [Acceso 19/05/2021].

[3] Representada en diferentes obras de Gustav Klimt, su labor principal consistió en el diseño de prendas vanguardistas para la sociedad vienesa de principios de siglo. Confeccionadas todas ellas junto con su hermana Helena, el salón de moda Schwestern Flöge diseñó prendas para clientes adinerados como Sonja Knips, Hermine Gallia o Eugenia Primaesi todas ellas mecenas de Klimt. Inspirándose en todo tipo de tejidos para la elaboración de sus colecciones, el fotógrafo Friedrich Walker retrato a una joven Emile vistiendo un jifu en su jardín de Villa Paulick en Seewalchen, reflejando un interés hacia todo tipo de objetos de los que poder nutrirse para la elaboración de sus colecciones https://www.gettyimages.es/fotos/emiliefl\%C3\%B6ge-fotos [Acceso 30/07/2021.

4] Fotografía del actor Cliffton Webb junto con las actrices Marlene Dietrich y Elizabeth Allan en 1935 vistiendo un abrigo jifu sin modificar. https://i.imgur.com/ag60jPP.jpg [Acceso 25/06/2021].

[5] Comunicación personal 6 de Octubre, 2020.
[6] EI MET conserva una chaqueta de los años 30 bajo el número de inventario 2009.300.8101. https://www.metmuseum.org/ art/collection/search/175126? searchField=All\&amp;sortBy=Re levance\&amp; $\mathrm{ft}=$ House + of + Chanel\&amp;offset=160\&amp;rpp =80\&amp:pos $=162$ [Acceso 29/01/2021].

[7] Kerry Taylor, 2010: https://www.invaluable.com/auction-lot/ an-imperial-dragon-robe-jifu-chinese-19th-44-c-3647dd3851 [Acceso 20/12/2020].

[8] Los ocho inmortales fueron seres legendarios extraídos del Taoísmo, los cuales conocían los secretos de la naturaleza. Estas deidades podían resucitar a los muertos, hacerse invisibles o convertir objetos inertes en preciados objetos valiosos, como el oro.

[9] Denominados como símbolos de la buena fortuna, todos ellos estaban relacionados con los emblemas reales asociados con Buda y sus creencias religiosas extraídas del Budismo.

[10] Comunicación personal, con Miki Komatsu Responsable del Departamento de Conservación y Restauración Textil del Asian Civilizations in Peranakan Museum (Singapur), 6 de Noviembre de 2020.

[11] El rapport es un término utilizado en el campo textil haciendo referencia al elemento mínimo de repetición utilizado para componer el motivo final a representar.

[12] Comunicación personal, 24 de Octubre, 2020.

[13] Diferentes objetos textiles fueron consultados durante este estudio como por ejemplo: T.433:60-1997, T.436:36-1997, T.436:75-1997

[14] Coco Chanel realizaría en 1929 varias prendas de estas características como la conservada en la colección del MET en Nueva York de colores rojos. Número de acceso 1984.31 a-c. https://www.metmuseum.org/art/collection/search/81483?s earchField=All\&amp; sortBy $=$ Relevance\&amp; $\mathrm{ft}=\mathrm{House}+\mathrm{of}+\mathrm{C}$ hanel\&amp;offset $=160 \&$ amp;rpp $=80 \&$ amp;pos $=190$ [Acceso 31/01/2021].

[15] Casas de moda francesas con un gran reconocimiento en los años 20 como Edgar et Marcel distribuyeron prendas similares como las conservadas hoy en la colección del KMDH, Número de inventario: K-108-1967 BC. https://www.sieradenmuze.nl/ collecties/namiddagensembles-ensembles-namiddagkledingvrouwenkleding [Acceso 31/01/2021].

[16] Obsérvese el tratamiento de conservación llevado a cabo en el jifu del Alfred Chester Beatty collection, donde justifican la razón de la inestabilidad de los bordados aplicados en las prendas jifu. https://chesterbeattyconservation.wordpress. com/tag/dragon-robes/. (Acceso 21/05/2021).

[17] Durante la investigación se pudieron registrar otro tipo de objetos textiles con presencia de láminas metálicas los cuales fueron intervenidos con adhesivos animales como la cola de 
pescado o cola de conejo. El National Research Institute of Cultural Heritage in South Korea publico en el año 2016 una serie de objetos textiles intervenidos con este tipo de adhesivos (National Research Institute of Cultural Heritage South Korea 2016: 152, 158, 190).

[18] Existen sin embargo, publicaciones que abordan la temática de limpieza de hilos metálicos sobre objetos textiles chinos, como el llevado a cabo por el Departamento de Conservación-Restauración Textil del MET en Nueva York junto con la Universidad Nacional de Korea en el año 2019. https:// www.e-jcs.org/journal/Table.php?xn=JCS-35-1-19.xml\&id= [Acceso 27/06/2021]

\section{Referencias}

BOUZARD, M. (1997). La Soierie Lyonnaise du 18 eme au 20 eme siecle. Dasles collections du musée des tissus de Lyon. Lyon: Editions Lyonnaises d'Art et d'Histoire.

BLOOMFIELD, N.J. (2020). Tackling a sticky situation: The examination and conservation of a Paul Poiret evening cloack. Master of Arts. New York: Proquest.

CARBONE, M.B. (2016). Conservation of a Han-Chinese Woman's skirt panel from the late nineteenth to early twentieth century. Master of Arts. New York: Proquest.

CHAN, H. (2017). "From costume to fashion. Visions of Chinese Modernity in Vogue Magazine, 1892-1943", Arts Orientalis, 47: 210-32. https://doi.org/10.3998/ars.13441566.0047.009

CHUNG YOUNG, Y. (1976). The origins and Historical Development of Embroidery in china, Japan, and Korea. Tesis Doctoral. New York University.

COPER HEWITT. "Skirt (China), 19 th century". Disponible en: https://collection.cooperhewitt.org/objects/18563133/ [Consulta: 05/02/2021].

DUSENBERRY M., M. (2004). Flowers, Dragons and Pine Trees: Asian Textiles in the collection of the Spencer Museum of Art. New York: Hudson Hills Press.

GARRET M., G. (1990). Mandarin Squares: Mandarins and their Insignia. Oxford: Oxford University Press

GARRET M., G. (1999). Chinese Dragon Robes. Oxford: Oxford University Press.

GARSIDE, P., WYETH, P. Y ZHANG, X. (2010). “Understanding the ageing behaviour of nineteenth and twentieth century tinweighted silks", Journal of the Institute of Conservation, 33: 2, 179-193. https://doi.org/10.1080/19455224.2010.501293

GILL, K. y BOERSMA, F (1997). "Solvent Reactivation of Hydroxypropyl Cellulose (Klucel G) in Textile Conservation", The Conservator, 21: 12-19. https://doi.org/10.1080/01410096.1997.
9995111 [Consulta: 23/05/2021]

HAN, J. (2016). The historical and chemical investigation of dyes in high status Chinese costume and textiles of the Ming and Qing Dynasties (1368-1911). Tesis Doctoral. School of Cultural and Creative Arts College of Arts, University of Glasgow.

HAN, J. y QUYE, A. (2018) "Dyes and Dyeing in the Ming and Qing Dynasties in China: Preliminary Evidence Based on Primary Sources of Documented Recipes", Textile History, 49(1): 44-70. https://doi.org/10.1080/00404969.2018.1440099

HACKE, M. (2009). "Weighted silk: history, analysis and conservation", Studies in Conservation, 54: 3-15. https://doi. org/10.1179/sic.2009.54.Supplement-1.3

HEROLDOVÁ, H. (2017). “Imperial Dragon in the roaring twenties: Qing Dynasty dress re-made", Annals of the Náprstek Museum, 38(1): 29-40. https://doi.org/10.1515/anpm-2017-0012

HEROLDOVÁ, H. (2016). "The Dragon Robe as the professional dress of the Qing Dynasty scholar-official (The Náprstek museum collection)", Annals of the Náprstek Museum , 37(2): 4972. https://doi.org/10.1515/anpm-2017-0012

HORSWILL, M. y YOUNG, A.R. (1992). "Characterization and Preservation of weighted silk", en Silk: Harper's Ferry Regional Textile Group, 11th Symposium, November 12-13, 1992, National Museum of American History.

HU, D. (2016). "The Techniques and Origin of Ornamental Gold Silks in Ancient China", Journal of Fiber Science and Technology, 72(6): 132-138. https://doi.org/10.2115/fiberst.2016-0020

HWEI-FE'N, C. (2015). Nyonya Needlework, Embroidery and Beadwork in the Peranakan World. Singapore: Asian Civilisations Museum.

INVALUABLE-Auction house (2010). "Lot 44: An Imperial dragon robe, jifu, Chinese, 19th". Disponible en: https://www.invaluable. com/auction-lot/an-imperial-dragon-robe-jifu-chinese-19th44-c-3647dd3851 [ Consulta: 20/12/2020].

LIN, S. Y DUARTE, C.J. (2017). “Uncovering the messages behind four Imperial Dragon Robes from Exhibitions with Yin and Yang Message", Journalism and Mass Communication, 17(1): 53-62. https://doi.org/10.17265/2160-6579/2017.01.006

LIN, S. (2013). "Booklet Imperial Costume Design: Yin-Yang Philosophical Influence". En Department of Family \& Consumer Sciences, University of Haway.

LUXFORD, N. (2009). Reducing the risk of open display: Optimising the preventive conservation of historic silks. Tesis Doctoral. University of Southampton. https://eprints.soton. ac.uk/162153/1/Naomi Luxford Thesis.pdf [Consulta: $16 / 03 / 2021]$

LUXFORD, N. (2011). "Non-destructive testing of silk: problems 
and possibilities". En ICOM Committee for Conservation 16th Triennial Meeting Lisbon Portugal 19-23. https://www. researchgate.net/publication/333565828 Non-destructive testing of silk problems and possibilities [Consulta: $16 / 03 / 2021]$

MUSÉE GALLIERA (2007). Les années folles 1919-1929. Paris: Paris Musees.

MILBANK, R.C. (2010). "Poiret, Paul". En The Berg Companion to Fashion, Valerie Steele (coord.). Oxford: Bloomsbury Academic. https://doi.org/10.1108/09504121111145492

MONTERO REDONDO, S. (2011). "La "seda cargada" en la indumentaria entre 1880 y 1930 . Metodología de estudio y propuesta de conservación-restauración", Ge-conservación, 2: 81-98. https://doi.org/10.37558/gec.v2i2.43

NATIONAL RESEARCH INSTITUTE OF CULTURAL HERITAGE SOUTH KOREA (2016). Conservation of Papers and Textiles. Gil Job le Media, Kindle edition.

NORD, Anders G., y TRONNER, K. (2000). 'A note on the Analysis of Gilded Metal Embroidery Threads', Studies in Conservation, 45(4): 274-279. https://doi.org/10.2307/1506864

SILBERSTEIN, R. (2020). A Fashionable Century. Textile Artistry and Commerce in the Late Qing. Seattle: University of Washington Press.

SIERADENMUZE. "Namiddagensembles ensembles namiddagkleding vrouwenkleding". Disponible en: https:// www.sieradenmuze.nl/collecties/namiddagensemblesensembles-namiddagkleding-vrouwenkleding [Consulta: 31/01/2021].

THE MET. "House of Chanel". Disponible en: https://www. metmuseum.org/art/collection/search/175126? searchField $=\mathrm{A}$ Il\&amp;sortBy=Relevance\&amp; $\mathrm{ft}=$ House+of+Chanel\&amp;of fset=160\&amp;rpp=80\&amp;pos=162 [Consulta: 29/01/2021].

TÍMÁR-BALÁZSY, A. y EASTOP, D. (2008). Chemical Principles of Textile Conservation. New York: Routledge.

TRETTER, S. Y WEINHÄUPL, P. (2016). Emile Flöge-Reforming fashion, inspiring art. Viena: Christian Brandsätter Verlag.

TURREL COLL, L.G. (2014). "Los tejidos coptos del Museo de Montserrat. Fuentes Documentales y formación de las colecciones". En La investigación textil y nuevos métodos de studio, Madrid:Fundación Lázaro Galdiano, 151-160.

UBACH, I. (2014). "Two figures from the Qing court at the Textile Museum and Documentation Centre", Datatextil, 31: 11-39. https://cdmt.cat/datatextil/?lang=es [Consulta: 25/06/2021]

VAINKER, S. (2004). Chinese Silk: A cultural history. New Brunswick: Rutgers University Press.
WILSON, K. J. (1990). “Powerful Form and Potent Symbol: The Dragon in Asia", Boletín del Cleveland Museum of Art, 77/8: 286323.

\section{Autor/es}

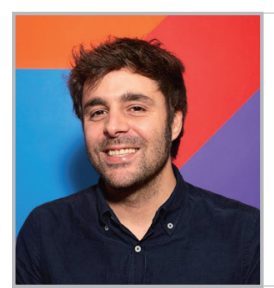

\section{César Rodríguez Salinas}

crodriguez@kunstmuseum.nl Departamento de Conservación y Restauración de Indumentaria y Tejidos Históricos del Kunstmuseum Den Haag http://orcid.org/0000-0002-9694-2275

Licenciado en Bellas Artes con la especialidad en Conservación y Restauración de Bienes Culturales Universidad del País Vasco (2011) y Máster Universitario en Técnicas de análisis aplicadas a la Conservación del Patrimonio Histórico Cultural Universidad Pablo de Olabide de Sevilla (2017). Inmerso en patrimonio textil e indumentaria histórica desde el 2011, ha trabajado en inumerables exposiciones de la mano de diferentes instituciones tanto nacionales como internacionales, entre otras el Museo Cristóbal Balenciaga de Getaria, el Museo de la Moda de Amberes o el Kunstmuseum Den Haag (KMDH). Desde el 2018 es Responsable del Departamento de Conservación y Restauración de Moda y Textiles Históricos del KMDH donde desempeña labores de conservación, restauración e investigación dentro de la colección. A través de estas colaboraciones con instituciones de patrimonio como el Instituto Valenciano de Conservación, Restauración e Investigación (IVCR+i) en España o el Rijksdienst voor het Cultureel Erfgoed (RCE) en los Países Bajos, se han podido publicar investigaciones como "Interdisciplinary research into the materials and degradation processes of an eighteenthcentury robe á la française" en el Journal of the Institute of Conservation o participar en congresos internacionales como el I Congreso sobre Balenciaga celebrado en España o el Dressing the Early Modern Network celebrado en Lisboa. En la actualidad, el departamento esta inmerso en la caracterización de tintes sintéticos y su efecto de degradación luminica en prendas de la colección datadas a finales del siglo XIX en colaboarción con el RCE y el IVCR+i.

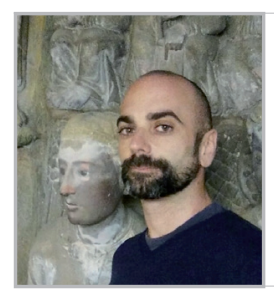
Livio Ferrazza
ferrazza_liv@gva.es
Conservador científico del Institut Valencià de Conservació, Restauració i Investigació $($ IVCR+i)
http://orcid.org/0000-0002-9694-2275

Livio Ferrazza es Doctor en Química y Diplomado en Ciencias para la conservación de bienes culturales por la Universidad "La Sapienza" de Roma. Desde 2007 colabora con el Laboratorio de Materiales del Instituto Valenciano de Conservación, Restauración e Investigación (IVCR+i), trabajando en los análisis de laboratorio de muestras de bienes culturales tales como pintura de caballete, pintura sobre tabla, pintura mural, piedra, textil, papel y metal. Ha participado en numerosas líneas de investigación con una destacada trayectoria en la evaluación 
tratamientos de estabilización y de limpieza de superficies policromadas, soporte pétreo y pinturas murales. Desde 2009 colabora con esta institución en los estudios analíticos y evaluación de los tratamientos de restauración en las pinturas murales de la Casa de Ariadna en Pompeya, en la Portada de Los Apóstoles de la Basílica Arciprestal de Morella (Castellón) o en la caracterización de materiales y evaluación de los tratamientos de limpieza en la pintura gótica valenciana como en el caso de la predela del Centenar de la Ploma del Victoria and Albert Museum de Londres. En 2014 recibe la beca Fundación Andrew W. Mellon colaborando con el Instituto del Patrimonio Cultural de España (IPCE) de Madrid en la evaluación de la eficacia y del riesgo asociado a los diferentes sistemas de estabilización y de limpieza de la policromía sobre piedra en el Pórtico de la Gloria de la Catedral de Santiago de Compostela. Ha colaborado en la línea de investigación del proyecto PNIC2015-05: Protocolo de evaluación del riesgo para la intervención en conjuntos escultóricos de piedra policromada, a través del cual se ha desarrollado un protocolo para la evaluación del riesgo y la eficacia en los diferentes tratamientos a emplear (limpieza, desbiotización, fijación, consolidación). Su participación en numerosos estudios químicos-analíticos aplicados a los bienes culturales han sido publicados y presentados en revistas y congresos especializados.

Artículo enviado el 22/02/2021 Artículo aceptado el 26/10/2021

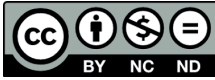

https://doi.org/10.37558/gec.v20i.980 